\title{
Community resilience: measuring a community's ability to withstand
}

\author{
M. Collins, J. Carlson \& F. Petit \\ Argonne National Laboratory, USA
}

\begin{abstract}
Several recent studies have provided a range of perspectives on the role of resilience in policies and programs designed to address natural and man-made threats. A review of those studies reveals that there is strong agreement that the concept of resilience must play a major role in assessing the extent to which various entities - critical infrastructure and key resources, systems, communities, and regions - are prepared to deal with the full range of threats they face. As resilience assessment methodologies continue to be developed and implemented for resilience at various levels, it is critical that a framework be developed to utilize measurements of resilience at multiple levels to characterize a community's resilience to potential hazards. Argonne National Laboratory, in partnership with the U.S. Department of Homeland Security, has developed a framework that combines existing measures of critical infrastructure and organization resilience with new measurement tools in order to characterize five subsystems of a community: the economy, physical infrastructure, government and nongovernmental organizations, emergency services, and the civilian population. The resilience of each of these subsystems will be measured through various tools, including an assessment of economic diversity; a review of community continuity of operations and government plans for essential functions; an analysis of critical infrastructure and organizational resilience to include lifeline utilities and vital private sector organizations; a study of disaster public education; communication and evacuation programs; and a review of the emergency services capabilities that a community possesses. Through the application of these measures, a community can better understand its current resilience posture, as well as implement a systematic approach to reduce vulnerabilities and consequences of potential hazards.
\end{abstract}

Keywords: resilience, community resilience, disaster preparedness, critical infrastructure resilience, risk mitigation, emergency management. 


\section{Introduction}

Several recent studies (see [1-8, 12], for example) have provided a range of perspectives on the role of resilience in policies and programs designed to address natural and man-made threats. A review of those studies reveals that there is strong agreement that the concept of resilience must play a major role in assessing the extent to which various entities - critical infrastructure and key resources, systems (e.g., electricity generation, transmission and distribution), communities, and regions - are prepared to deal with the full range of threats they face.

Agreement regarding the importance of resilience notwithstanding, there is considerable disagreement over a number of issues associated with the concept. These include how resilience should be defined, whether it is an outcome or a process, and the type of resilience a particular analysis is considering [4, 5].

This paper has two primary objectives. The first is to develop a clear distinction between resilience as it relates to critical infrastructure and community resilience. The second is to develop a framework for constructing a measure of community resilience that can be combined with existing measures of asset readiness to provide a quantitative assessment of risk mitigation across different assets and levels of aggregation (e.g., systems, communities, regions).

\section{Resilience defined}

As noted in the introduction, there is no single, universally agreed-upon definition of resilience. Instead, its definition and measurement vary with respect to type (e.g., economic, engineering, ecological) and focus (e.g., community [societal], critical infrastructure, supply chain). When focusing on resilience as it relates to managing risks attributable to natural or man-made threats, one of the most significant disagreements concerns the question of whether resistance (i.e., protection) should be included as a component of resilience. As part of their analysis of community resilience, Norris et al [3] completed a review of the literature on resilience. On the basis of their review, they concluded that

"[r]esilience has been defined in a variety of ways.... Most definitions emphasize a capacity for successful adaptation in the face of disturbance, stress, or adversity. Although there are exceptions, most discussions, if not the definitions themselves, distinguish resilience from 'resistance"” (p. 129).

The authors provided a summary of the definitions of resilience found in 21 of the articles they reviewed. Of those 21 definitions, 16 define resilience as capacities/actions that occur after some type of disturbance, stress, or adverse event has occurred. Consistent with the findings of Norris et al [3], several of the studies we reviewed [1, 3, 5, 7, 13 (citing [2])] describe resilience as the ability of an entity to recover, or "bounce back," from the adverse effects of a natural or man-made threat. These definitions take the position, either explicitly or 
implicitly, that protection and resilience are distinct elements of an overall strategy to address such threats.

In contrast, the U.S. Department of Homeland Security (DHS) [9], the Homeland Security Studies and Analysis Institute (HSI) [5], and the National Science and Technology Council's Subcommittee on Disaster Reduction (SDR) [10] explicitly include resistance in their definitions of resilience.

\subsection{Reconciling different definitions}

Regarding the question of whether resistance should be included in the assessment of resilience, Cutter et al [4] argue that:

More recent research on resilience from a homeland security perspective (primarily protecting critical infrastructure from terrorism) [5] also focuses on critical infrastructure resilience assuming that resilience is an outcome measure with an end goal of limiting damage to infrastructure (termed resistance); mitigating the consequences (called absorption); and recovery to the pre-event state (termed restoration). While perhaps useful for counterterrorism and protection of critical infrastructure, this operational framework ignores the dynamic social nature of communities and the process of enhancing and fostering resilience within and between communities (p. 2).

There are two important points here. First, defining resilience to include resistance reflects a view that resilience is an outcome, as opposed to a process. Second, the appropriate definition of resilience is context dependent. In this case, one could argue that the definition offered by DHS and Kahan et al [5] is appropriate when attention is focused on CIKR. In contrast, when the focus is on community resilience, a definition that focuses on adaptation and recovery, and explicitly ignores resistance, is preferred.

\subsubsection{Critical infrastructure resilience versus community resilience}

The justification for the distinction between resilience at the asset level versus the community level rests on the following points. First, limiting our focus to terrorist threats, experience to date suggests that an attack will focus on a specific asset or collection of assets - buildings, buses, trains, airplanes. In this scenario, protection is more appropriately addressed by the individual assets (i.e., critical infrastructure) that are the likely target of an attack. (Even if we think in terms of a system, ultimately protection must be addressed by the individual elements of the system, e.g., generation, distribution, transmission, and independent system operators in the electricity sector.) The notion that communities should engage in protective measures would invariably require that they focus on individual assets and systems; however, the owners/managers of individual assets are much better positioned to identify the protective measures currently in place and to evaluate the incremental value of additional protective measures. As such, communities would make better use of limited resources by leaving this aspect of risk management to the individual assets. 
Second, focusing on the time dimension of a threat, protection/resistance consists of actions that are taken prior to a threat's occurrence either to forestall it or to reduce (mitigate) its adverse effects. Once the threat has been "realized" in the form of a successful attack, attention shifts to the ability of the affected entity-asset, system, community, region-to maintain functions, absorb impacts, adapt response(s), degrade in a graceful manner as opposed to abruptly, recover functions, and restore operations. Here again, it makes sense to include some consideration of protection in the critical infrastructure-level measure of resilience. This approach is taken because the level of protection the asset has put into place prior to an event will undoubtedly influence the level of damage ultimately sustained and, consequently, the asset's ability to recover and maintain its core functions. This ability at the asset level will, in turn, feed into the extent of the community's overall ability to absorb, adapt to, and recover from the adverse effects of the attack.

Risk management at the community level requires consideration of both the risks faced by CIKR and the community's ability to respond, adapt to, and recover from a disruptive event. The latter can be captured by measuring the community's resilience. The next two sections of this paper address two related issues: (1) the definition of community resilience, and (2) how to measure community resilience.

\section{Community resilience}

Argonne National Laboratory (Argonne) [11] has developed a methodology that can be used to measure the resilience of critical infrastructure. This measure is appropriate when assessing risk and risk mitigation efforts directed at specific critical infrastructure assets. If, however, the focus is on communities, a different measure of resilience is needed. Our review of the literature yielded four recent studies-Norris et al [3], Stewart et al [8], Longstaff et al [6], and Cutter et al [4] - that specifically address the determinants of community resilience. Of these, the latter three studies address the measurement of community resilience as well.

The study by Norris et al [3] presents a comprehensive review of the literature on resilience and develops a model of how community resilience is determined. According to Norris et al [3], "[c]ommunity resilience is a process linking a network of adaptive capacities (resources with dynamic attributes) to adaptation after a disturbance or adversity" (p. 127). In addition, "resilience rests on both the resources themselves and the dynamic attributes of those resources ...we use the term 'adaptive capacities' to capture this combination" (p. 135). The dynamic attributes (properties) of resilience resources include robustness, redundancy, and rapidity. Norris et al [3] also identify four sets of "networked resources" that are responsible for determining community resilience: economic development, social capital, information and communication, and community competence.

The authors use their model of community resilience to identify a set of actions they believe will enhance community resilience to disasters. The set of 
actions includes: (1) developing economic resources, reducing risk and resource inequalities, and addressing areas of greatest social vulnerability; (2) meaningfully engaging local people in every step of the mitigation process so as to access social capital; (3) developing organizational networks and relationships that will rapidly mobilize emergency and ongoing support services in the event of a disaster; (4) engaging in interventions that boost and protect naturally occurring social supports in the aftermath of disasters; and (5) exercising flexibility and focusing on building effective and trusted information and communication resources that function in the face of unknowns.

While adopting the definition of resilience offered by Norris et al [3], Stewart et al [8] identify supply chain resilience, critical infrastructure resilience, economic resilience, social resilience, and public-private partnerships (PPPs) as the primary determinants of community resilience. In their model, the resilience of relevant supply chains and the critical infrastructure that exist in the community affect one another and, in conjunction with the community's economic and social resilience, determine the level of community resilience. In addition, the relationships that government (public) agencies develop with private sector partners (i.e., PPPs), can positively influence supply chain and critical infrastructure resilience.

Longstaff et al [6] define community resilience as "the ability of a community to absorb a disturbance while retaining its essential functions" (p. 4). In the authors' model, community resilience is a function of resource robustness and adaptive capacity. Resource robustness is measured by resource performance, redundancy, and diversity. Adaptive capacity is measured by institutional memory, innovative learning, and connectedness. Community resilience is determined by the resource robustness and adaptive capacity of each of five key community subsystems: ecological, economic, civil society, governance, and physical infrastructure.

A recent study by Cutter et al [4] provides a framework for assessment of community resilience and then applies that framework to the counties in the Federal Emergency Management Agency's Region IV. Although the authors do not offer a formal definition of community resilience, their approach explicitly focuses on the ability to respond to and recover from a threat once it has been realized. On the basis of a theoretical model, the authors include the following five categories in their measure of community resilience: social resilience, economic resilience, institutional resilience, infrastructure resilience, and community capital resilience.

\subsection{Comparison/contrast of the four studies}

Focusing first on the definition employed in each study, there is relatively little difference (practically speaking) among the four studies. All four tend to focus on how the community responds after a threat has been realized; that is, protection/resistance is not included in any of the definitions. There is considerably more variation, however, among the studies regarding the primary determinants of community resilience. Taken together, the four studies identify 14 different components that are assumed to affect community resilience. As the 
following summary of definitions and possible measures shows, however, there is overlap among the individual components.

Economic resilience: Economic resilience is the only component included in all four of the models considered herein. There is also a fair amount of agreement regarding the variables that Stewart et al [8], Longstaff et al [6], and Cutter et al [4] suggest ought to be included in the measurement of economic resilience (e.g., employment statistics, income equality, labor market conditions, and business diversification).

Civil society/Community capital resilience/Community competence/Social capital/Social resilience: In comparing the definitions of civil society in Longstaff et al [6], community capital resilience in Cutter et al [4], community competence and social capital in Norris et al [3], and social resilience in Stewart et al [8], some degree of overlap is suggested. This impression is reinforced when we consider the specific measures of resilience for these components as suggested in each study.

Ecological resilience: The study by Longstaff et al [6] is the only one that includes ecological resilience in its measure of community resilience. Their suggested measures of ecological resilience include water supplies, wind patterns, climate, soil quality, topography, diversity of habitats, agricultural diversity, how quickly key elements of the local environment can regenerate in the event of a disaster, and the ability of the environment to support a diversity of crops and wildlife.

Governance resilience/Institutional resilience: The study by Longstaff et al [6] includes governance resilience in its measure of community resilience. There is some overlap between governance resilience and institutional resilience, which Cutter et al [4] include in their model. For example, Longstaff et al [6] refer to the connectedness of the various units of government in times of disruption, while Cutter etal [4] focus on political fragmentation. In addition, Longstaff et al [6] point to the cost and quality of services delivered in relation to the resources collected from citizens, while Cutter et al [4] suggest including the percentage of municipal expenditures that are apportioned for fire, police, and emergency medical services.

Critical infrastructure/Physical infrastructure/Infrastructure: There is a high degree of overlap as it relates to the definitions of critical infrastructure resilience in Stewart et al [8] and physical infrastructure in Longstaff et al [6]. However, there is considerably less overlap between the definitions proposed by these two and the measurement of infrastructure proposed by Cutter et al [4].

Public-private partnerships: The study by Stewart et al [8] is the only one to explicitly model PPPs as a determinant of community resilience. PPPs enter the model through their effects on critical infrastructure and supply chain resilience. Longstaff et al [6] do, however, refer to PPPs in their definition of governance resilience.

Supply chain resilience: The study by Stewart et al [8] is the only one to explicitly include supply chain resilience in its list of the determinants of community resilience. The authors' suggested measures of supply chain 
resilience include redundancy, flexibility, density, complexity, node criticality, and public-private partnerships.

\section{Calculating a community resilience index}

In this section, we propose a process for measuring community resilience. To do that, we must first establish a working definition of community resilience and specify a model that explains how community resilience is determined.

\subsection{A working definition of community resilience}

As discussed in Section 2 of this paper, a review of the general concept of resilience reveals that most of the disagreement among competing definitions concerns whether protection/resistance should be included as an element of resilience. That being said, this issue appears to arise only with respect to the general concept of resilience. Our review of analyses of community resilience revealed no such disagreement; none of the four studies reviewed in this paper includes protection/resistance in their definitions of resilience. The definitions include the following:

- Norris et al [3]/Stewart et al [8]: Community resilience is a process linking a network of adaptive capacities (resources with dynamic attributes) to adaptation after a disturbance or adversity.

- Longstaff et al [6]: Community resilience is the ability of a community to absorb a disturbance while retaining its essential functions.

- Cutter et al [4]: Their definition focuses on the ability to respond to and recover from a threat once it has been realized.

The key terms employed in these definitions include absorb, adapt, respond, and recover. While one might be tempted to think of these elements in a sequential fashion (e.g., first absorb, then respond/adapt, and finally recover), we argue that actions associated with each of these elements of resilience are interdependent and can occur simultaneously. Based on the preceding discussion, we define community resilience as "the ability of a community to absorb, respond/adapt to, and recover from a disturbance while retaining its essential functions."

\subsection{Determinants of community resilience}

The next step is to specify a model that identifies the primary determinants of community resilience. Referring again to the studies reviewed in Section 3, community resilience is a function of some set of subsystems or networked resources. For purposes of comparison, we assume that the terms "subsystem" and "set of networked resources" are functionally equivalent. Each of the four models considered herein can be summarized as follows:

- Norris et al [3] view community resilience as a function of the resilience of four sets of networked resources-economic development, social capital, 
information and communication, and community competence-which, in turn, determine community resilience.

- Stewart et al [8] view community resilience as a function of supply chain resilience, critical infrastructure resilience, economic resilience, social resilience, and PPPs.

- Longstaff et al [6] view community resilience as a function of the resilience of five subsystems-ecological, economic, civil society, governance, and physical infrastructure.

- Cutter et al [4] include the following five categories in their measure of community resilience: social resilience, economic resilience, institutional resilience, infrastructure resilience, and community capital resilience.

Taken together, subsystems that might contribute to the assessment of community resilience include: economic resilience, ecological resilience, critical infrastructure/physical infrastructure/infrastructure resilience, governance/institutional resilience, civil society/community capital/community competence/social capital/social resilience, information and communication resilience, and supply chain resilience.

The model we propose to use to measure community resilience is adapted from the list of subsystems listed above. According to our model, community resilience is a function of the resilience of the following subsystems: the community's economy, critical infrastructure (selected components), governmental and nongovernmental services (institutions), emergency services sector, and the civilian population. The justification for each of the components included in our model is as follows.

- Economic resilience: Economic resilience is included in all four models reviewed herein and clearly has important implications for the ability of a community to "bounce back" from a disturbance, such as a terrorist attack.

- Infrastructure resilience: In our model, infrastructure resilience is a function of a subset of critical infrastructure sectors (Commercial Facilities, Communications, Information Technology, Energy, Healthcare and Public Health, Transportation Systems, and Water). These specific sectors were selected on the basis of their relationship to such concepts as adaptation and recovery of a community once a threat has been realized.

- Institutional resilience: The ability of governmental or nongovernmental units to continue to function in the event of a disturbance will obviously have a profound impact on the community's ability to absorb, respond to, and recover from the disturbance. This view is reflected in the considerable emphasis that DHS has placed on the development of continuity of operations plans and continuity of government plans at all levels.

- Emergency services sector resilience: Emergency services will also have a large impact on the community's ability to absorb, respond to, and recover from a disturbance, and this subsystem encompasses information and communication resilience as called for by Norris et al [3].

- Civilian population resilience: In Section 3.1, we argue that civil society, community capital resilience, community competence, social capital, and social resilience are roughly similar concepts that focus, to varying degrees, 
on the ability of a community's general public to respond to a disturbance. It should be clear that the public's inability to adapt, respond to, and recover from a disturbance will seriously limit the community's ability to bounce back, regardless of the resiliency of the other subsystems included here. Thus, our model includes a measure of the resilience of the community's civilian population.

Depending on whether the measurement of community resilience focuses on man-made or naturally occurring hazards, we may include measures of supply chain and/or ecological resilience as well.

\subsection{Determinants of resilience of subsystems}

The next step in this process is to identify properties/characteristics that can be used to assess the resilience of each of the subsystems included in our model. The studies by Norris et al [3], Longstaff et al [6], and Argonne [11] offer guidance in this regard. Norris et al [3] use the properties of robustness, redundancy, and rapidity to assess the resilience of networked resources. Longstaff et al [6] use the properties of resource robustness and adaptive capacity to assess the resilience of subsystems. Argonne [11] uses the properties of robustness, resourcefulness, and rapid recovery to assess critical infrastructure resilience.

The key terms listed above-robustness, redundancy, resourcefulness, rapidity, recovery, and adaptive capacity — are defined as follows:

- Robustness: Norris et al [3] define robustness as the ability to withstand stress without suffering degradation, while Argonne [11] defines it as the ability to maintain critical operations and functions in the face of crisis. Regarding measures of robustness, Longstaff et al [6] cite resource performance, redundancy, and diversity, whereas Argonne [11] focuses on redundancy, prevention/mitigation, and the ability to maintain key functions.

- Redundancy: Norris et al [3] define redundancy as the extent to which elements are substitutable in the event of disruption or degradation. Comparing the three approaches, however, we believe it is more appropriate to designate redundancy as a determinant of robustness as is suggested by Longstaff et al [6], National Infrastructure Advisory Council [7], and Argonne [11].

- Resourcefulness: Norris et al [3] define resourcefulness as the capacity to identify problems and mobilize resources. Argonne [11] defines resourcefulness as the ability to skillfully prepare for, respond to, and manage a crisis and suggests several possible measures of resourcefulness, including the conduct of training exercises and the existence of stockpiles, protective measures, alternative sites, awareness, new resources, and response capabilities.

- Rapidity: Norris et al [3] defines rapidity as the speed with which a resource can be accessed and used (includes the property of resourcefulness).

- Rapid Recovery: Argonne [11] defines rapid recovery as the ability to return to and/or reconstitute normal operations as quickly and efficiently as possible 
after a disruption and suggests coordination and restoration as possible measures.

- Adaptive Capacity: According to Longstaff et al [6], adaptive capacity "is a function of the ability of individuals and groups to: (1) store and remember experiences; (2) use that memory and experience to learn, innovate, and reorganize resources in order to adapt to changing environmental demands; and (3) connect with others inside and outside the community to communicate experiences and lessons learned, self-organize or reorganize in the absence of direction, or to obtain resources from outside sources" (p. 7). The authors also cite possible measures of adaptive capacity, including institutional memory, innovative learning, and connectedness.

In our model, the resilience of each subsystem is a function of robustness, resourcefulness, rapid recovery, and adaptive capacity.

\subsection{Quantifying community resilience}

Regarding the quantification of resilience, we envision constructing a set of indices that measure the resilience of each of the subsystems included in our model of community resilience. Indices of this type are referred to as composite indicators. According to Cutter et al [4]:

[a] composite indicator is the mathematical combination of individual variables or thematic sets of variables that represent different dimensions of a concept that cannot be fully captured by any individual indicator alone. Composite indicators are increasingly recognized as useful tools for policy making and public communication because they convey information that may be utilized as performance measures (pp. 2-3).

The specific indices include the following:

- Economic Resilience Index: Using the properties/characteristics described in the preceding section, a set of variables will be identified that can be used to produce an Economic Resilience Index (ERI). This index will capture the ability of the vital economic components of a community to survive disruptions and/or quickly stand back up to maintain the economic viability of a given community.

- Infrastructure Resilience Index: The Resilience Index (RI) developed by Argonne [11] will be used to measure the resilience of individual sectors, which could be segregated to life-line utilities: Communications, Information Technology, Energy, Healthcare and Public Health, Transportation Systems, and Water. The individual RIs will then be rolled up into a single Infrastructure Resilience Index (IRI) that will portray the overall resilience of the infrastructure located within and servicing the community.

- Institutional Resilience Index: In order to capture the resilience of the individual governmental and nongovernmental organizations that provide vital functions to the community, an index measuring the specific framework of the organizations combined with a review of necessary continuity of 
operations and continuity of government (COOP/COG) plans will be developed.

- Emergency Services Sector Capabilities Index: The Emergency Services Sector Capability Index (ESSCI), developed by Argonne, focuses on the various dimensions of emergency services, including emergency medical services, law enforcement, fire services, search and rescue, explosive threat response, hazardous materials response, emergency management, and response and coordination capabilities, as well as the ability to communicate with and inform the public (thus capturing information and communication resilience as called for by Norris et al [3]). This index will be utilized to characterize the ability of a given community to protect its citizens, infrastructure, and organizations from possible hazards; mitigate potential impacts; quickly and efficiently respond once an event occurs; and then organize long-term recovery procedures.

- Public Preparedness Index: Utilizing the Public Preparedness Index (PPI) currently in production at Argonne National Laboratory, a review will be conducted of a community's public emergency preparedness programs, warning systems, and crisis communication methods, as well as other methods of preparing and caring for individuals before, during, and after an event.

Once the individual indices_ERI, RI, IRI, COOP/COG Review, ESSCI, and PPI-have been applied to a given community, they will be presented individually rather than being rolled up into a single measure. Creating a single metric would mask a great deal of information and would reduce the ability to analyze different possible combinations of resilience across the five subsystems. In particular, examining each of the indices individually and in relation to one another will provide decision makers with a more detailed picture of a community's current resilience posture. In addition, it will facilitate identification of those areas in which investment of additional resources to bolster resilience is likely to yield the greatest return per dollar spent.

\section{Summary}

In order to better understand the ability of individual communities to absorb, respond/adapt to, and recover from a disturbance while retaining their essential functions, emergency planners must understand the resilience attributes of the subsystems that make up a community. In order to do so, we have identified five subsystems that characterize the citizens of a community, the services provided to the residents of the community, and the infrastructure-economic and otherwise - that make the community a viable option for current or future residents. Having identified those subsystems, we have also developed separate measures of each (i.e., the indices described in Section 4.4) to help communities characterize their abilities to withstand and recover from a potential incident or attack. Gaining a better understanding of each of the components enables a more thorough analysis of the strengths and weaknesses of a community in terms of resilience, as well as a prioritized list of options to consider when contemplating 
how best to improve overall community resilience. The effort to improve community resilience does not guarantee that incidents will not occur, nor does such an effort guarantee that if incidents do occur, the community will be able to avoid serious impacts. However, it does allow a community to be proactive in reframing its programs, services, and organizations in a way to better prepare for potential hazards.

\section{References}

[1] Boin, A., \& McConnell, A., Preparing for critical infrastructure breakdowns: the limits of crisis management and the need for resilience, Journal of Contingencies and Crisis Management, 15(1), pp. 50-59, 2007.

[2] Allenby, B. \& Fink, J., Toward inherently secure and resilient societies, Science, 309(5737), pp. 1034-1036, 2005.

[3] Norris, F.H., Stevens, S.P., Pfefferbaum, B., Wyche, K.F. \& Pfefferbaum, R.L., Community resilience as a metaphor, theory, set of capacities, and strategy for disaster readiness, American Journal of Community Psychology, 41(1), pp. 127-150, 2008.

[4] Cutter, S.L., Burton, C.G. \& Emrich, C.Y., Disaster resilience indicators for benchmarking baseline conditions, Journal of Homeland Security and Emergency Management, 7(1), pp. 1-22, 2010.

[5] Kahan, J.H., Allen, A.C. \& George, J.K., An operational framework for resilience, Journal of Homeland Security and Emergency Management, 6(1), pp. 1-48, 2009, www.bepress.com/jhsem/vol6/iss1/83.

[6] Longstaff, P.H., Armstrong, N.J., Perrin, K.A., Parker, W.M. \& Hidek, M., Community Resilience: A Function of Resources and Adaptability, Institute for National Security and Counterterrorism, Syracuse University, June 2010.

[7] National Infrastructure Advisory Council (NIAC), Critical Infrastructure Resilience, Final Report and Recommendations, U.S. Department of Homeland Security, Washington DC, 2009, www.dhs.gov/xlibrary/assets/ niac/niac critical infrastructure resilience.pdf.

[8] Stewart, G.T., Kolluru, R. \& Smith, M., Leveraging public-private partnerships to improve community resilience in times of disaster, International Journal of Physical Distribution \& Logistics Management, 39(5), pp. 343-364, 2009.

[9] U.S. Department of Homeland Security, Risk Steering Committee; DHS Risk Lexicon, Washington DC, 2008.

[10] Subcommittee on Disaster Reduction (SDR), Grand Challenges for Disaster Reduction, National Science and Technology Council, Washington DC, 2005.

[11] Argonne National Laboratory, Constructing a Resilience Index for the Enhanced Critical Infrastructure Protection Program, Argonne National Laboratory, Decision and Information Sciences Division, ANL/DIS-10-9, Argonne IL, USA, 2010. 
[12] U.S. Department of Homeland Security, National Infrastructure Protection Plan: Partnering to Enhance Protection and Resiliency, Washington DC, 2009. Online. www.dhs.gov/xlibrary/assets/NIPP Plan.pdf.

[13] Homeland Security Advisory Council (HSAC), Report of the Critical Infrastructure Task Force, U.S. Department of Homeland Security, Washington DC, 2006, www.dhs.gov/xlibrary/assets/ HSAC_CITF_Report_v2.pdf. 